\title{
The Growth Hormone Secretagogue Hexarelin Protects Rat Cardiomyocytes From in vivo Ischemia/Reperfusion Injury Through Interleukin-1 Signaling Pathway
}

\author{
Jiannan HuANG, ${ }^{1,2}$ MD, Yi LI, ${ }^{3}$ MD, Juan ZHANG, ${ }^{2}$ MD, \\ Yusheng LiU, ${ }^{1} \mathrm{MD}$, and Qinghua Lu, ${ }^{1} \mathrm{MD}$
}

\begin{abstract}
SUMMARY
Hexarelin, a synthetic growth hormone-releasing peptide, has been proven to possess cardioprotective actions through its binding to the growth hormone secretagogue receptor (GHSR) 1a and the non-GHSR receptor CD36. However, its effect on myocardial ischemia/reperfusion (I/R) injury has not been fully clarified in vivo. We aimed to determine whether hexarelin treatment could protect cardiomyocytes from I/R injury and to examine the underlying mechanisms. In vivo hearts of male SD rats underwent 30 minutes of ischemia by left coronary artery ligation followed by reperfusion. The rats were then treated subcutaneously twice daily with hexarelin [100 $\mu \mathrm{g} / \mathrm{kg} \cdot \mathrm{day}]$, ghrelin [400 $\mu \mathrm{g} /$ $\mathrm{kg} \cdot \mathrm{day}]$, or saline for 7 days. Echocardiography, malondialdehyde detection, and histochemical staining were performed after treatment. In addition, Western blot was used to examine the expression levels of IL-1 $\beta$, IL-1Ra, and IL-1RI. Our study showed that hexarelin treatment improved cardiac systolic function, decreased malondialdehyde production, and increased the number of surviving cardiomyocytes. The beneficial effects of hexarelin treatment were slightly superior to those of equimolar ghrelin treatment. We meanwhile confirmed that hexarelin induced down-regulation of IL- $1 \beta$ expression and up-regulation of IL-1Ra expression in I/R myocardium, which could be neutralized by the GHSR antagonist [D-Lys3]-growth hormone releasing peptide-6 ([D-Lys3]-GHRP-6). These findings suggest that hexarelin protects in vivo cardiomyocytes from I/R injury partly by modification of the IL-1 signaling pathway through the activation of cardiac GHSR1a receptors. (Int Heart J 2017; 58: 257-263)
\end{abstract}

Key words: Malondialdehyde, Oxidative stress, Apoptosis, Inflammation, Echocardiography, Masson stain, Western blot analysis

$\mathrm{A}$ cute myocardial infarction, which is caused by a sudden embolism of the coronary arteries, is a leading cause of mortality. As the primary clinical therapy for treatment of myocardial infarction, myocardial reperfusion can also aggravate cardiac injury. ${ }^{1)}$ Its mechanism includes calcium overload, cardiomyocyte apoptosis, and reactive oxygen species (ROS) accumulation. ${ }^{2-5)}$ Finding a strategy to alleviate myocardial I/R injury has become a hot research field in cardiology.

In vivo $\mathrm{I} / \mathrm{R}$ models can cause regional myocardial $\mathrm{I} / \mathrm{R}$ injury by ligating the left anterior descending coronary artery, which have been widely used to mimic the process of myocardial infarction and subsequent reperfusion. ${ }^{6)}$ Compared to the in vitro global $\mathrm{I} / \mathrm{R}$ models, in vivo $\mathrm{I} / \mathrm{R}$ models can not only reveal changes in hemodynamics, and cardiac structure and function after $\mathrm{I} / \mathrm{R}$, but also display the impact of molecules in reperfusion flow on I/R myocardium, such as ROS and inflammation cytokines. ${ }^{7)}$ The in vivo I/R models are more consistent with the pathophysiological process of myocardial I/R injury.

Ghrelin, a growth hormone-releasing peptide produced in the stomach, is an endogenous ligand of growth hormone secretagogue receptor (GHSR) 1a. Previous studies have demonstrated the cardioprotective effects of exogenous ghrelin administration. ${ }^{8)}$ Hexarelin, a synthetic analog of ghrelin, shows effects similar to those of ghrelin and is more chemically stable, making it a potential alternative to ghrelin. Its beneficial action on the cardiovascular system has been reported by a lot of research, including inhibition of cardiomyocyte apoptosis, ${ }^{9-11)}$ anti-atherosclerosis, ${ }^{12,13)}$ improving cardiac output, ${ }^{14-17)}$ and suppressing cardiac fibrosis. ${ }^{18)}$ These effects are considered to be mediated not only by GHSR1a but also by activation of cardiac CD36 receptors. ${ }^{19)}$ The existence of cardiac GHSR1a and CD36 receptors indicates hexarelin has direct cardiovascular effects independent of growth hormone $(\mathrm{GH})$ release and neuroendocrine stimulation, which was also proved by several studies. ${ }^{8)}$ [D-Lys3]-growth hormone releasing peptide-6 ([D-Lys3]-GHRP-6), a GHSR1a antagonist, can block the effects of both ghrelin and hexarelin. ${ }^{1,18,20)}$

Interleukin-1 (IL-1), which was originally known as "endogenous pyrogen", was one of the first inflammatory cy-

From the ${ }^{1}$ Department of Cardiology, The Second Hospital of Shandong University, Jinan, ${ }^{2}$ Department of Cardiology, The Central Hospital of Zibo City, and ${ }^{3}$ Obstetric Genetic Disease Laboratory, Maternal and Child Health Hospital of Zibo City, Zibo, Shandong Province, China.

Address for correspondence: Qinghua Lu, MD, Department of Cardiology, The Second Hospital of Shandong University, 247 Benguan Avenue, Jinan, Shandong, 250033, China. E-mail: lqhzhy@sina.com

Received for publication May 20, 2016. Revised and accepted July 13, 2016

Released in advance online on J-STAGE March 17, 2017.

All rights reserved by the International Heart Journal Association. 
tokines to be discovered. ${ }^{21)}$ It consists of two distinct ligands, $\mathrm{IL}-1 \alpha$ and $\mathrm{IL}-1 \beta$, and they possess a high degree of sequence homology and approximately the same biological activity. ${ }^{22)}$ IL- $1 \alpha$ and IL- $1 \beta$ play their biological roles by binding to two kinds of receptors, IL-1RI and IL-1RII. ${ }^{23)} \mathrm{IL}-1$ receptor antagonist (IL-1Ra), which naturally exists in many kinds of tissues, can block the IL-1 signaling pathway by competitively binding to IL-1RI. ${ }^{24)} \mathrm{IL}-1 \mathrm{Ra}$ has been shown to have a cardioprotective function. $^{25-28)}$

Although the cardioprotective effects of hexarelin have been partially revealed by many studies, the action of hexarelin on myocardial I/R injury in vivo have not been fully confirmed, whereas the underlying mechanisms remain unknown. In our study, using rat models of myocardial I/R, we attempted to observe the effects of hexarelin treatment on myocardial I/R injury, as well as clarify the role of the IL-1 signaling pathway in the cardiovascular action of hexarelin.

\section{Methods}

All experiments were approved by the Animal Ethics Committee of Shandong University. Pathogen-free, 6-weekold, male SD rats (SLACCAS, Shanghai, China) were maintained in air-filtered units at $21 \pm 2{ }^{\circ} \mathrm{C}$ and $50 \pm 15 \%$ relative humidity. Rats were provided with sterile water and rodent food.

Animal models of myocardial $\mathbf{I} / \mathbf{R}$ injury: In vivo $\mathrm{I} / \mathrm{R}$ models were established as described previously. ${ }^{29)}$ All rats were anesthetized with isoflurane (induced at $4 \%$, maintained at $1.5 \%$ ) before surgery. They were then intubated and supported by an animal ventilator. After a left thoracotomy, the left anterior descending coronary artery was ligated by a 6- 0 prolene suture 3 $\mathrm{mm}$ from its origin. After 30 minutes of occlusion, the suture was released for reperfusion. The chest was then closed and the rats were placed on a heating blanket to recover from the anesthesia. In the sham group, we only opened and closed the chest without performing any other operation.

Animal grouping and administration: The I/R model rats were administered ghrelin (400 $\mu \mathrm{g} / \mathrm{kg} \cdot$ day, $n=15)$, hexarelin (100 $\mu \mathrm{g} / \mathrm{kg} \cdot$ day, $n=20$ ), or saline (same volume, $n=15$ ) by subcutaneous bolus injection in the neck from 30 minutes before surgery and then daily until 7 days after I/R injury. In addition, in order to be used for Western blot analysis, another 10 I/R model rats were administered [D-Lys3]-GHRP-6 alone (100 nmol/kg.day, $n=5)$ or together with hexarelin $(100 \mu \mathrm{g} /$ $\mathrm{kg} \cdot \mathrm{day}, n=5$ ) as described above. The 20 rats in the sham group were given hexarelin $(100 \mu \mathrm{g} / \mathrm{kg} \cdot \mathrm{day}, n=10)$ or saline (same volume, $n=10$ ).

Measurement of body weight, heart weight, blood pressure, and heart rate: The body weight, mean arterial pressure, and heart rate of each rat were recorded before and 7 days after the operation. After the rats were sacrificed and the hearts removed, heart weight was recorded. An automatic sphygmomanometer with a tail-cuff pressure transducer (BP-98A, Softron, Tokyo) was used to measure the blood pressure and heart rate. Each value was the average of 3 consecutive measurements.

Malondialdehyde detection: ROS accumulation was been measured in I/R myocardium using biochemical assays of lipid peroxidation products. Malondialdehyde is a marker of lipid peroxidation and ROS activity since ROS degrade polyunsatu- rated lipids and form malondialdehyde. ${ }^{30)}$ Cardiac tissue samples were homogenized in $10 \%$ Tris-buffered saline at $4^{\circ} \mathrm{C}$ with a homogenizer. Homogenates were then centrifuged at $500 \times \mathrm{g}$ for 15 minutes. The supernatants were collected and evaluated using the malondialdehyde assay kit (Jiancheng Bioengineering Institute, Nanjing, China) according to the manufacturer's protocol.

Echocardiography: Echocardiographic studies were performed as described previously, ${ }^{31)}$ using a high-resolution ultrasound system for rodents (Vevo770, VisualSonics Inc, Ontario, Canada) under anesthesia with $1.5 \%$ isoflurane 7 days after establishment of the animal models. To assess the function of the left ventricles, left ventricular end-diastolic diameter (LVDd), LV anterior wall thickness (LVAWth), and ejection fraction (LVEF) were calculated. Before establishment of the animal models, the rats did not show any differences in the basal values of these parameters (data not shown).

Histological examination: After the echocardiographic studies, the rats were sacrificed and the hearts were removed and divided from the apex to the base into two equal transverse sections. The apex part was fixed in $10 \%$ formalin, dehydrated through an upgraded ethanol series, and embedded in paraffin. Paraffin sections were prepared with Masson's trichrome staining for further measurement. Slices were mounted and photographed. We measured the area of the viable myocardium for each slice using Image-Pro Plus Version 6 (Installshield Software Corporation). The myocardial survival rate is presented as a percentage of the total heart tissue. The average of all slices was calculated to compare between the groups.

Western blot analysis: Heart tissues were pooled for each experimental condition and homogenized. Equal amounts of protein sample (100 ug) were separated by SDS-PAGE and electrotransferred to PVDF membranes. After blocking, the membrane was incubated with a 1:1000 dilution of polyclonal rabbit anti-rat IL-1 $\beta$ (Santa Cruz Biotechnology, Dallas, TX, USA) primary antibodies, polyclonal rabbit anti-rat IL-1Ra (Santa Cruz Biotechnology) primary antibodies, or polyclonal rabbit anti-rat IL-1RI (Santa Cruz Biotechnology) primary antibodies overnight at $4^{\circ} \mathrm{C}$, followed by extensive washes with Tris buffered saline supplemented with $0.1 \%$ Tween-20. After hybridization with a 1:1000 dilution of goat radish-peroxidaseconjugated secondary antibody (Beyotime Institute of Biotechnology, Jiangsu, China) for 1 hour, the immunoreactive proteins were detected using an ECL Western blotting detection system (Beyotime Institute of Biotechnology). GAPDH was used as the internal control.

Statistical analysis: All data are expressed as the mean \pm standard deviation (SD). Differences among the groups of rats were analyzed by one-way ANOVA for multiple comparisons, followed by the two-tailed Student $t$-test. In all comparisons, the differences were considered to be statistically significant at a value of $P<0.05$.

\section{Results}

Hexarelin treatment did not lead to significant changes in body weight, heart weight, blood pressure, or heart rate: As shown in the Table, there were no significant differences between groups in the baseline values of body weight, blood pressure, and heart rate. After 7 days of ghrelin treatment, body weight 
Table. Effects of Hexarelin on Body Weight, Heart Weight, Blood Pressure, and Heart Rate

\begin{tabular}{lccccc}
\hline & $\begin{array}{c}\text { Sham }+ \\
\text { Saline }\end{array}$ & $\begin{array}{c}\text { Sham }+ \\
\text { Hexarelin }\end{array}$ & $\begin{array}{c}\text { I/R+ } \\
\text { Saline }\end{array}$ & $\begin{array}{c}\text { I/R+ } \\
\text { Ghrelin }\end{array}$ & $\begin{array}{c}\text { I/R+ } \\
\text { Hexarelin }\end{array}$ \\
\hline BW at baseline $(\mathrm{g})$ & $202 \pm 11$ & $206 \pm 12$ & $200 \pm 7$ & $208 \pm 11$ & $206 \pm 15$ \\
BW after 7 days of treatment $(\mathrm{g})$ & $254 \pm 16$ & $264 \pm 14$ & $247 \pm 10$ & $275 \pm 12^{\#}$ & $257 \pm 13$ \\
Heart weight/BW $(\mathrm{g} / \mathrm{kg})$ & $3.25 \pm 0.11$ & $3.21 \pm 0.15$ & $3.5 \pm 0.2^{*}$ & $3.42 \pm 0.17$ & $3.38 \pm 0.19$ \\
MAP at baseline (mmHg) & $110 \pm 7$ & $107 \pm 5$ & $112 \pm 5$ & $109 \pm 4$ & $112 \pm 9$ \\
MAP after 7 days of treatment (mmHg) & $109 \pm 8$ & $105 \pm 7$ & $119 \pm 8$ & $117 \pm 9$ & $115 \pm 4$ \\
HR at baseline & $362 \pm 9$ & $367 \pm 12$ & $359 \pm 13$ & $364 \pm 10$ & $365 \pm 7$ \\
HR after 7 days of treatment & $360 \pm 7$ & $369 \pm 11$ & $357 \pm 10$ & $362 \pm 9$ & $367 \pm 14$ \\
\hline
\end{tabular}

Results are shown as the mean \pm SD. ${ }^{*} P<0.05$ versus sham+saline group; ${ }^{\#} P<0.05$ versus $\mathrm{I} / \mathrm{R}+$ saline group. BW indicates body weight; MAP, mean arterial pressure; and HR, heart rate.

had increased compared to the saline-treated rats, while other indicators showed no significant changes. Hexarelin treatment did not lead to significant changes in body weight, heart weight, blood pressure, or heart rate in either sham-operated or $\mathrm{I} / \mathrm{R}$ rats.

Hexarelin protects cardiac tissue from malondialdehyde elevation after myocardial I/R injury: Malondialdehyde was detected 7 days after myocardial I/R injury (Figure 1). There was a significant increase in the malondialdehyde level in heart tissues of saline-treated I/R rats compared to sham-operated rats. With ghrelin or hexarelin treatment after myocardial I/R injury, the density of malondialdehyde in cardiac tissue decreased, indicating that both ghrelin and hexarelin protect cardiac tissue from malondialdehyde elevation after myocardial I/R injury. There were no significant differences in malondialdehyde levels between ghrelin-treated and hexarelin-treated I/R rats. Furthermore, hexarelin treatment had no apparent influence on sham-operated rats.

Hexarelin increases the number of viable myocardial cells after myocardial I/R injury: Tissue sections of the sham+saline group and sham+hexarelin group appeared to have a normal cardiac structure, and no cell injury or necrosis was observed (Figure 2A, 2B). In the I/R+saline group, marked fibrous scar tissue had formed and there were only a few viable myocardial cells within the scar tissues (Figure 2C). Compared to the I/ $\mathrm{R}+$ saline group, myocardial injury and fibrosis in the $\mathrm{I} /$ $\mathrm{R}+$ hexarelin group were significantly alleviated, and the number of viable myocardial cells increased significantly (Figure $2 \mathrm{E}$ ), which was also observed in the $\mathrm{I} / \mathrm{R}+$ ghrelin group (Figure 2D). We further calculated the percentage of the area of viable myocardium in cardiac tissue in order to accurately compare the differences in cell survival among these groups. As shown in Figure 2F, the percentage of the area of viable myocardium was elevated in the $\mathrm{I} / \mathrm{R}+$ ghrelin and $\mathrm{I} /$ $\mathrm{R}+$ hexarelin groups compared to the $\mathrm{I} / \mathrm{R}+$ saline group. In addition, the myocardial survival rate of the $\mathrm{I} / \mathrm{R}+$ hexarelin group was higher than that of the I/R+ghrelin group. The results indicated that both ghrelin and hexarelin treatment promoted cell survival of I/R myocardium, and that hexarelin was even more effective than ghrelin.

Hexarelin improves cardiac function after myocardial I/R injury: To evaluate cardiac systolic function in rats from different groups, LVEF, LVDd and LVAWth were measured by an echocardiographic examination 7 days after the operation (Figure 3). Compared to the sham+saline group, the I/R+saline group had significantly lower LVEF, larger LVDd, and smaller LVAWth. In the I/R+ghrelin group, the LVEF was higher than

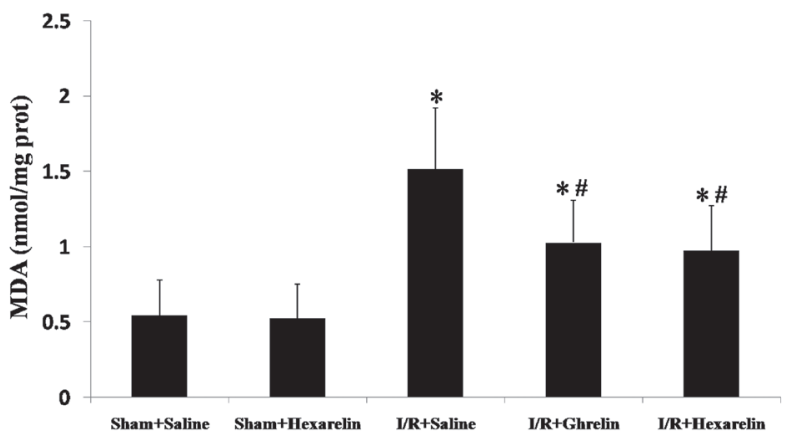

Figure 1. Effect of hexarelin on density of malondialdehyde in heart tissues. The malondialdehyde level in the $\mathrm{I} / \mathrm{R}+$ saline group $(n=15)$ was significantly higher than that in the sham+saline group $(n=10)$ and sham+hexarelin group $(n=10)$. Compared to the I/R+saline group, the malondialdehyde level decreased significantly in the I/R+ghrelin group ( $n$ $=15)$ and $\mathrm{I} / \mathrm{R}+$ hexarelin group $(n=20)$. Results are shown as the mean \pm SD. ${ }^{*} P<0.05$ versus sham + saline group; ${ }^{\#} P<0.05$ versus $\mathrm{I} / \mathrm{R}+$ saline group. MDA indicates malondialdehyde.

that in the I/R+saline group, but the other two parameters did not change significantly. With hexarelin treatment, changes in LVEF and LVDd obviously improved compared to the I/ $\mathrm{R}+$ saline group, and the LVEF values were even higher than in ghrelin-treated rats. Hexarelin treatment had no apparent influence on the sham-operated rats.

Role of IL-1 signaling pathway and GHSR1a in the cardioprotective effects of hexarelin after I/R injury: Protein expressions of IL-1 $\beta$, IL-1RI, and IL-1Ra in cardiac tissue 7 days after treatment were examined by Western blot analysis. As shown in Figure 4, IL-1 $\beta$ expression increased and IL-1Ra expression decreased significantly after I/R, and hexarelin treatment inhibited the changes in the IL-1 signaling pathway in I/R myocardium. In order to investigate the action of GHSR1a on hexarelin-induced IL-1 signaling pathway alternation in I/R myocardium, the GHSR1a antagonist [D-Lys3]-GHRP-6 was used alone or with hexarelin in I/R rats. Our studies showed [D-Lys3]-GHRP-6 alone had no effect on IL-1 $\beta$ and IL-1Ra expressions, but it could partly block the effect of hexarelin treatment on the IL-1 signaling pathway. No significant differences were detected in IL-1RI expression between the groups and hexarelin treatment showed no apparent effect on shamoperated rats. 
A

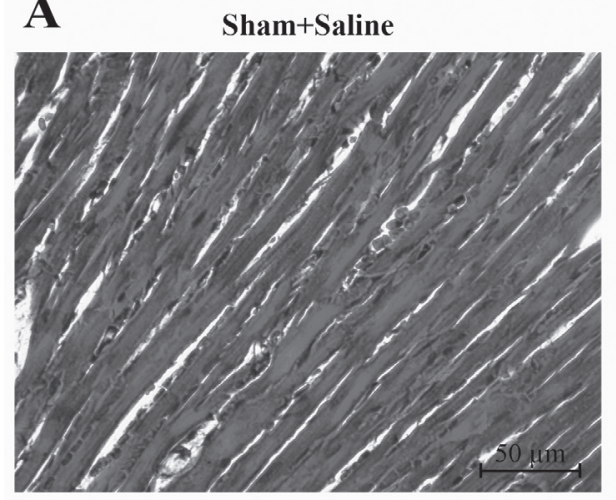

C

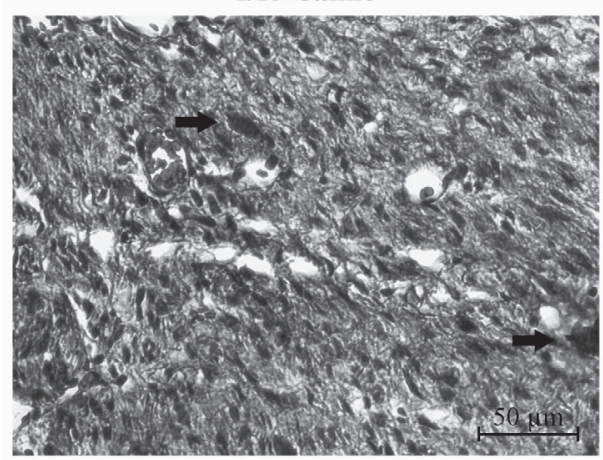

$\mathbf{E}$

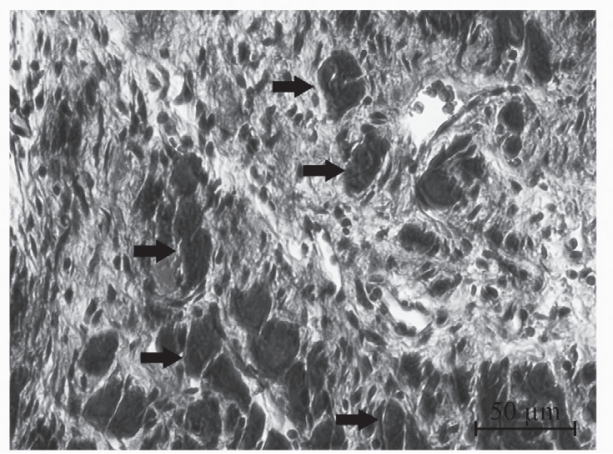

B

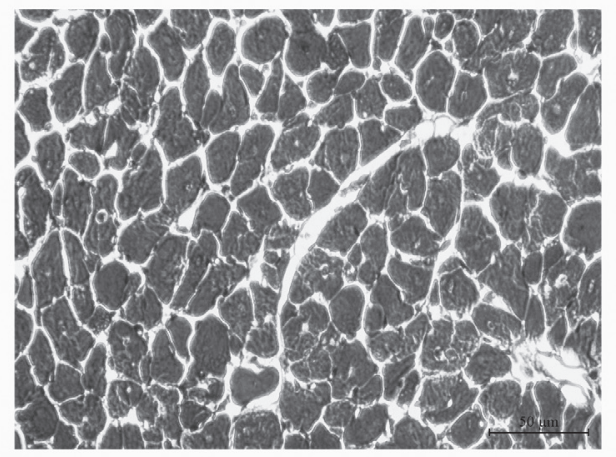

D

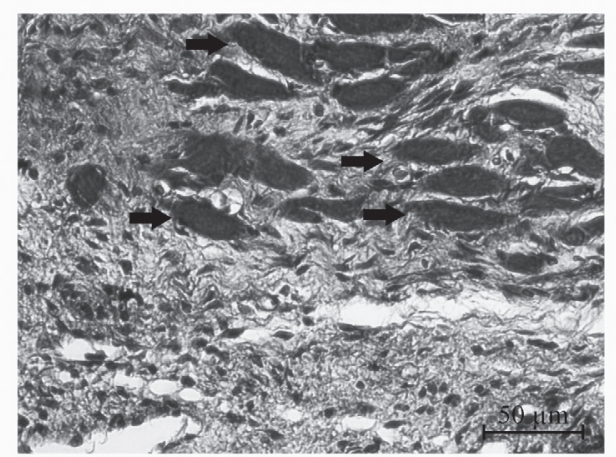

F

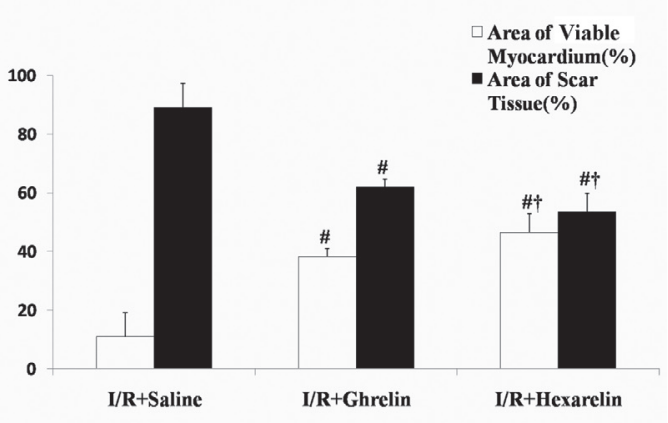

Figure 2. Effect of hexarelin on histological changes after myocardial I/R injury. The figure shows the slice images of cardiac tissue with Masson's trichrome staining (400x) 7 days after I/R (A, B, C, D, E) and normalized ratio of survival myocardium with IPP software $(\mathbf{F})$. The viable myocardium was stained red and marked with an arrow. Compared to the I/ $\mathrm{R}+$ saline group $(n=10)$, the number of viable cardiomyocytes increased in the $\mathrm{I} / \mathrm{R}+$ ghrelin group $(n=15)$ and increased more significantly in the $\mathrm{I} / \mathrm{R}+$ hexarelin group $(n=15)$. Results are shown as the mean $\pm \mathrm{SD} .{ }^{*} P<0.05$ versus $\mathrm{I} / \mathrm{R}+$ saline group; ${ }^{\dagger} P<0.05$ versus $\mathrm{I} / \mathrm{R}+$ ghrelin group.

\section{DisCUSSION}

In the present study, we have confirmed that hexarelin produces a protective effect on cardiomyocytes exposed to in vivo I/R injury. We have also discovered that hexarelin treatment leads to down-regulation of IL- $1 \beta$ expression and up-regulation of IL-1Ra expression in I/R myocardium. We have demonstrated for the first time that the IL-1 signaling pathway participates in the cardioprotective effects of hexarelin on $I / R$ myocardium and that hexarelin-induced IL-1 signaling pathway alternation is mediated by cardiac GHSR1a receptors.

The mechanisms of myocardial I/R injury are multifaceted, including ATP depletion, $\mathrm{Ca}^{2+}$ overload, and ROS generation. Several lines of evidence suggest that ROS play an important role in the pathogenesis of myocardial I/R injury, including myocardial stunning, cardiomyocyte apoptosis, and reperfusion arrhythmias. ${ }^{2-5)}$ Potential sources of ROS production during $\mathrm{I} / \mathrm{R}$ have been identified in myocytes, vascular en- 
A
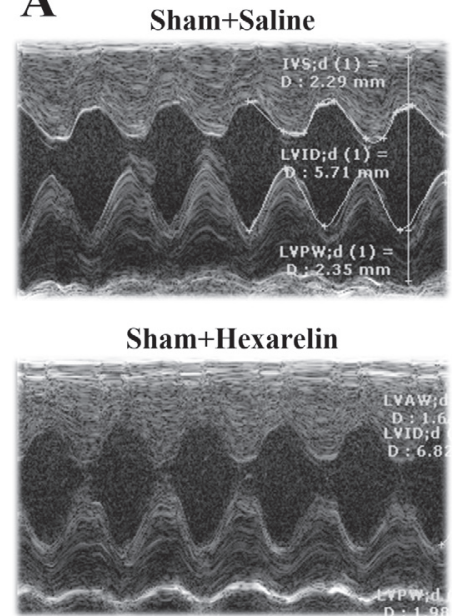

$\mathbf{I} / \mathbf{R}+$ Saline

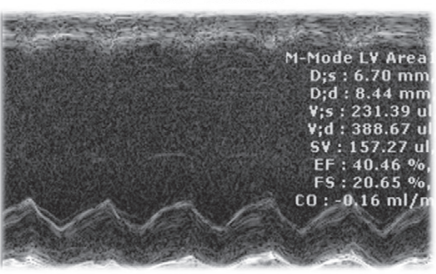

I/R+Ghrelin

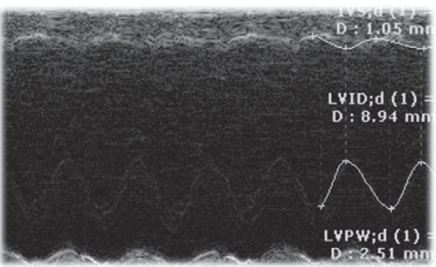

I/R+Hexarelin

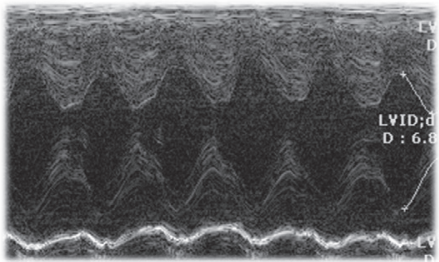

B

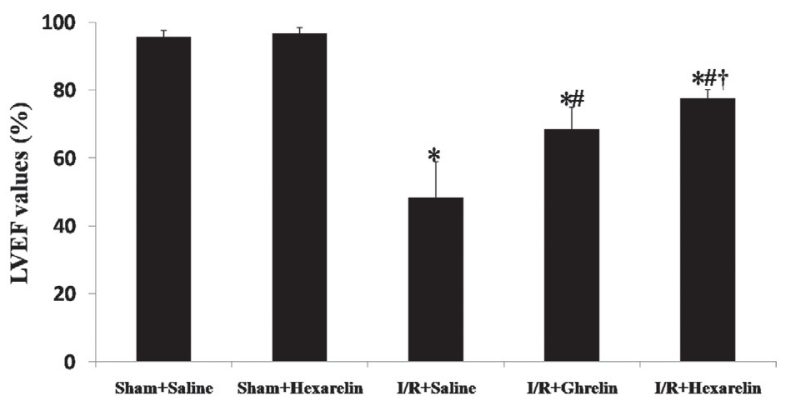

C

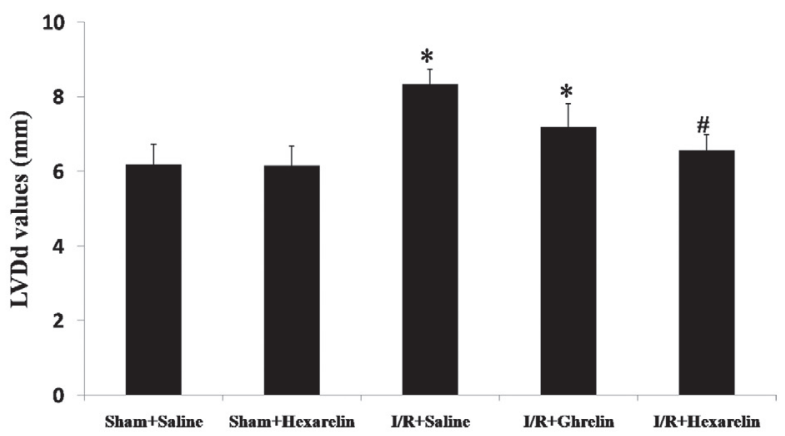

D

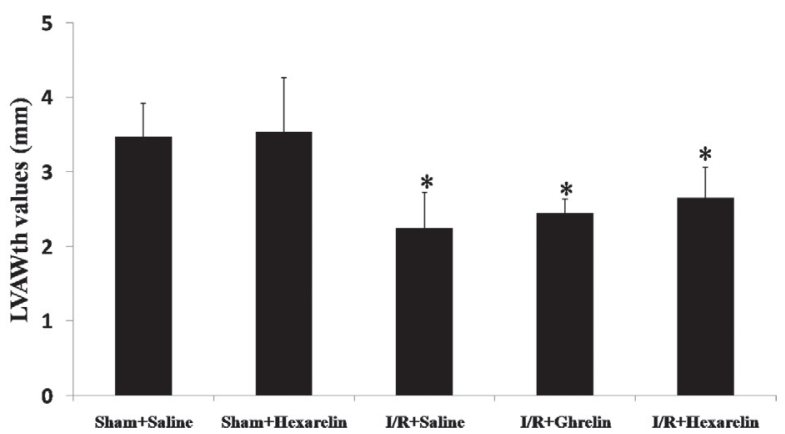

Figure 3. Effect of hexarelin on cardiac function after I/R injury. The figure shows representative echocardiograms (A) and comparison of LVEF (B), LVDd (C), and LVAWth (D) between groups. The value was acquired 7 days after I/R. In each group, $n=10$. Results are shown as the mean $\pm \mathrm{SD}$. ${ }^{*} P<0.05$ versus sham+saline group; ${ }^{\sharp} P<0.05$ versus $\mathrm{I} / \mathrm{R}+$ saline group; ${ }^{\top} P<0.05$ versus $\mathrm{I} / \mathrm{R}+$ ghrelin group. $\mathrm{LVEF}$ indicates left ventricular ejection fraction; $\mathrm{LVDd}, \mathrm{LV}$ end-diastolic diameter; and LVAWth, LV anterior wall thickness.

dothelium, and leukocytes. Neutrophils have been proven to be the main sources. ${ }^{32)} \mathrm{IL}-1$, which is a proinflammation cytokine, is released during ischemia, and recruits neutrophils to the myocardium after reperfusion together with other cytokines and complements. Neutrophils are then activated and interact with endothelial cells, which may be a critical event that initiates the inflammatory component of reperfusion injury. ${ }^{23)}$ This means the IL-1 signaling pathway participates in the process of ROS production through the recruitment and activation of neutrophils.

Apoptosis of cardiomyocytes has been confirmed to be involved in the process of myocardial damage after I/R injury. ${ }^{33-35)}$ It has been demonstrated that IL- $1 \beta$ activates AP- 1 and the NF- $\kappa \mathrm{B}$ system, induces changes in the gene transcription of Bak and Bcl-x, and then promotes apoptosis of cardiomyocytes. ${ }^{36)}$ In addition to apoptosis, I/R injury-mediated inflammation can also lead to aggravation of myocardial damage. Numerous experiments have shown that the IL-1 signaling pathway participates in the inflammatory responses of myocardial I/R injury. ${ }^{37)}$ Furthermore, IL-1 regulates phenotype variation and fibroblast activity, as well as the activation of MMPs/ TIMPs, leading to changes in the degradation of extracellular 
A

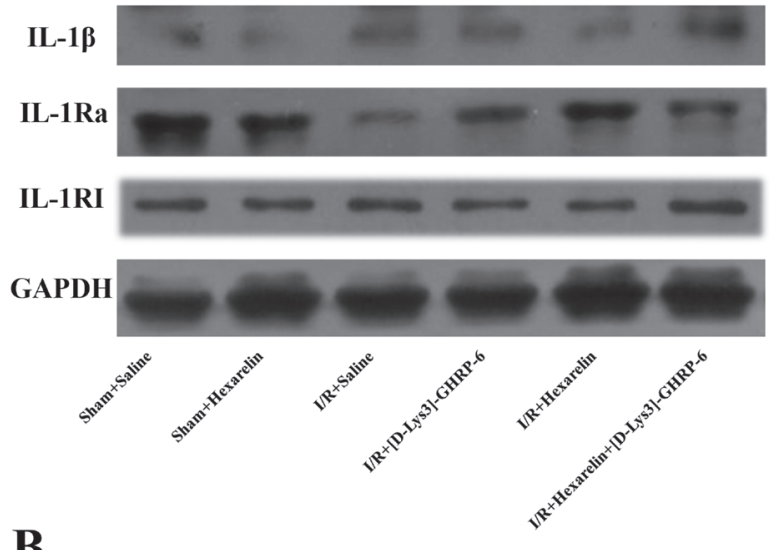

B
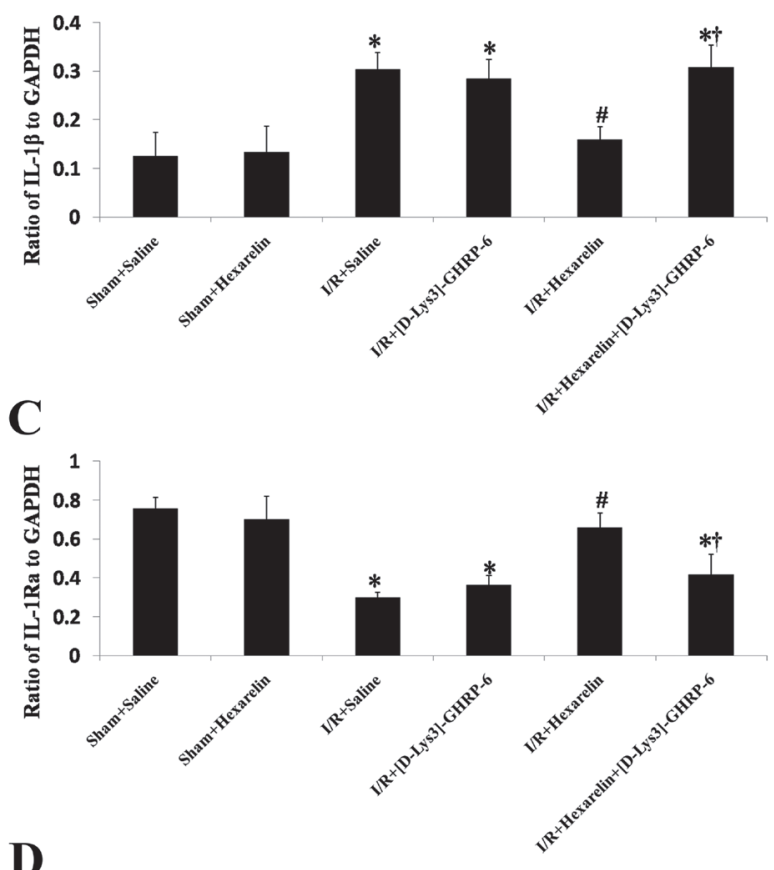

D

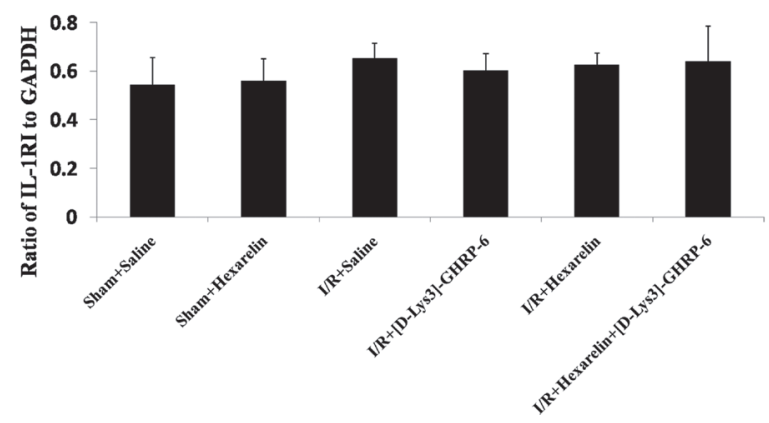

Figure 4. Effect of hexarelin treatment on IL-1 signaling pathway. The figure shows representative Western blots for target proteins and GAPDH (A), followed by comparison of changes in expressions of IL-1 $\beta$ (B), IL1Ra (C), and IL-1RI (D). In each group, $n=5$. Results are shown as the mean \pm SD. ${ }^{*} P<0.05$ versus sham + saline group; ${ }^{\#} P<0.05$ versus $\mathrm{I} /$ $\mathrm{R}+$ saline group; ${ }^{\dagger} \mathrm{P}<0.05$ versus $\mathrm{I} / \mathrm{R}+$ hexarelin group. matrix and deposition of collagen. ${ }^{38,39)}$

Cardiac systolic dysfunction after I/R injury presents as low cardiac output and dilated left ventricular volume. Myocardial necrosis and apoptosis after ischemia lead to a reduction in the number of viable cardiomyocytes, which ultimately aggravates cardiac dysfunction. Some cytokines released during I/R inhibit the contractility of cardiomyocytes as well. It has been shown that exogenous IL- $1 \beta$ can directly inhibit myocardial contractility through the NO dependent pathway as well as decrease the cAMP concentration. ${ }^{37}$ Previous studies on Anakinra, a synthetic inhibitor of IL- $1 \beta$, have demonstrated that it can improve cardiac performance after I/R injury. ${ }^{27)}$ The IL-1 signaling pathway has also been found to play an important role in the development of congestive heart failure and cardiac remodeling. ${ }^{40)}$

In the present study, we used malondialdehyde detection to assess the ROS production in I/R myocardium, histochemical detection to assess the number of viable cardiomyocytes after $\mathrm{I} / \mathrm{R}$, and echocardiography to assess cardiac function in the rats. The results showed that hexarelin had a comprehensive protective effect on $\mathrm{I} / \mathrm{R}$ myocardium, presenting as a significant improvement of the above mentioned parameters. Previous studies have indicated that the cardiovascular action of hexarelin occurs through the activation of two cardiac receptors, GSHR1a receptors and CD36 receptors. ${ }^{19)}$ However, the changes in intracellular signaling pathways and gene expressions induced by receptor activation have not been fully clarified. In our study, we observed down-regulation of IL- $1 \beta$ expression and up-regulation of IL-1Ra expression in I/R myocardium after hexarelin treatment, which could be interrupted by the GHSR1a antagonist [D-Lys3]-GHRP-6. In view of the fact that the cardiovascular effect of the IL-1 signaling pathway has been confirmed by numerous studies as discussed above, hexarelin-induced modification of the IL-1 signaling pathway through activating cardiac GHSR1a receptors can explain the protective effect of hexarelin treatment on I/R myocardium. Nevertheless, the limit of our research is that we have not made clear the action of cardiac CD36 receptors on alternation of the IL-1 signaling pathway induced by hexarelin.

In conclusion, this study has demonstrated that hexarelin treatment can protect in vivo heart from I/R injury, represented as a lower malondialdehyde level, more viable cardiomyocytes, and better cardiac function. Our study further revealed that hexarelin exerted its cardioprotective action partly through modification of the IL-1 signaling pathway, which might be an outcome of GHSR1a activation. As a stable and effective GH secretgogue, hexarelin may be a potential target for the treatment of myocardial I/R injury.

\section{Disclosure}

Conflict of interest: There is no conflict of interest in this study.

\section{REFERENCES}

1. Ma Y, Zhang L, Edwards JN, Launikonis BS, Chen C. Growth hormone secretagogues protect mouse cardiomyocytes from in vitro ischemia/reperfusion injury through regulation of intracellu- 
lar calcium. PLoS One 2012; 7: e35265.

2. Murphy E, Steenbergen C. Mechanisms underlying acute protection from cardiac ischemia-reperfusion injury. Physiol Rev 2008; 88: 581-609. (Review)

3. Bell RM, Yellon DM. There is more to life than revascularization: therapeutic targeting of myocardial ischemia/reperfusion injury. Cardiovasc Ther 2011; 29: e67-79. (Review)

4. Ibanez B, Cimmino G, Badimon JJ. Myocardial reperfusion injury. N Engl J Med 2007; 357: 2409.

5. Sanada S, Komuro I, Kitakaze M. Pathophysiology of myocardial reperfusion injury: preconditioning, postconditioning, and translational aspects of protective measures. Am J Physiol Heart Circ Physiol 2011; 301: H1723-41. (Review)

6. Ma Y, Zhang L, Launikonis BS, Chen C. Growth hormone secretagogues preserve the electrophysiological properties of mouse cardiomyocytes isolated from in vitro ischemia/reperfusion heart Endocrinology 2012; 153: 5480-90.

7. Furman E, Acad BA, Sonn J, Raul A, Kedem J. Effect of global vs regional ischaemia upon myocardial contractility and oxygen balance. Cardiovascular Research 1985; 19: 606-12.

8. Mao Y, Tokudome T, Kishimoto I. The cardiovascular action of hexarelin. J Geriatr Cardiol 2014; 11: 253-8. (Review)

9. Pang JJ, Xu RK, Xu XB, et al. Hexarelin protects rat cardiomyocytes from angiotensin II-induced apoptosis in vitro. Am J Physiol Heart Circ Physiol 2004; 286: H1063-9.

10. Filigheddu N, Fubini A, Baldanzi G, et al. Hexarelin protects $\mathrm{H} 9 \mathrm{c} 2$ cardiomyocytes from doxorubicin-induced cell death. Endocrine 2001; 14: 113-9.

11. Xu XB, Pang JJ, Cao JM, et al. GH-releasing peptides improve cardiac dysfunction and cachexia and suppress stress-related hormones and cardiomyocyte apoptosis in rats with heart failure. Am J Physiol Heart Circ Physiol 2005; 289: H1643-51.

12. De Gennaro-Colonna V, Rossoni G, Cocchi D, Rigamonti AE, Berti F, Muller EE. Endocrine, metabolic and cardioprotective effects of hexarelin in obese Zucker rats. J Endocrinol 2000; 166: 529-36.

13. Pang J, Xu Q, Xu X, et al. Hexarelin suppresses high lipid diet and vitamin D3-induced atherosclerosis in the rat. Peptides 2010; 31: 630-8.

14. Broglio F, Guarracino F, Benso A, et al. Effects of acute hexarelin administration on cardiac performance in patients with coronary artery disease during by-pass surgery. Eur J Pharmacol 2002; 448: 193-200.

15. Sun Q, Ma Y, Zhang L, Zhao YF, Zang WJ, Chen C. Effects of GH secretagogues on contractility and $\mathrm{Ca} 2+$ homeostasis of isolated adult rat ventricular myocytes. Endocrinology 2010; 151: 4446-54.

16. Frascarelli S, Ghelardoni S, Ronca-Testoni S, Zucchi R. Effect of ghrelin and synthetic growth hormone secretagogues in normal and ischemic rat heart. Basic Res Cardiol 2003; 98: 401-5.

17. Tivesten A, Bollano E, Caidahl K, et al. The growth hormone secretagogue hexarelin improves cardiac function in rats after experimental myocardial infarction. Endocrinology 2000; 141: 60-6.

18. Xu X, Pang J, Yin H, et al. Hexarelin suppresses cardiac fibroblast proliferation and collagen synthesis in rat. Am J Physiol 2007; 293: H2952-8.

19. Mao Y, Tokudome T, Kishimoto I, et al. Hexarelin treatment in male ghrelin knockout mice after myocardial infarction. Endocrinology 2013; 154: 3847-54.

20. Xu X, Ding F, Pang J, et al. Chronic administration of hexarelin attenuates cardiac fibrosis in the spontaneously hypertensive rat. Am J Physiol Heart Circ Physiol 2012; 303: H703-11.

21. Dinarello CA. Cytokines as endogenous pyrogens. J Infect Dis 1999; 179: S294-304. (Review)

22. Allan SM, Tyrrell PJ, Rothwell NJ. Interleukin-1 and neuronal in- jury. Nat Rev Immunol 2005; 5: 629-40. (Review)

23. Bujak M, Frangogiannis NG. The role of IL-1 in the pathogenesis of heart disease. Arch Immunol Ther Exp (Warsz) 2009; 57: 16576. (Review)

24. Dinarello CA. The role of the interleukin-1-receptor antagonist in blocking inflammation mediated by interleukin-1. N Engl J Med 2000; 343: 732-4. (Review)

25. Abbate A, Salloum FN, Vecile E, et al. Anakinra, a recombinant human interleukin-1 receptor antagonist, inhibits apoptosis in experimental acute myocardial infarction. Circulation 2008; 117: 2670-83.

26. Salloum FN, Chau V, Varma A, et al. Anakinra in experimental acute myocardial infarction--does dosage or duration of treatment matter? Cardiovasc Drugs Ther 2009; 23: 129-35.

27. Ikonomidis I, Lekakis JP, Nikolaou M, et al. Inhibition of interleukin-1 by anakinra improves vascular and left ventricular function in patients with rheumatoid arthritis. Circulation 2008; 117: 2662-9.

28. Abbate A, Kontos MC, Grizzard JD, et al. Interleukin-1 blockade with anakinra to prevent adverse cardiac remodeling after acute myocardial infarction (Virginia Commonwealth University Anakinra Remodeling Trial [VCU-ART] Pilot Study). Am J Cardiol 2010; 105: 1371-7.

29. Tarnavski O. Mouse surgical models in cardiovascular research. Methods Mol Biol 2009; 573: 115-37. (Review)

30. Del Rio D, Stewart AJ, Pellegrini N. A review of recent studies on malondialdehyde as toxic molecule and biological marker of oxidative stress. Nutr Metab Cardiovasc Dis 2005; 15: 316-28. (Review)

31. Kuroda R, Shintani-Ishida K, Unuma K, Yoshida K. Immobilization stress with $\alpha 2$-adrenergic stimulation induces regional and transient reduction of cardiac contraction through Gi coupling in rats. Int Heart J 2015; 56: 815-24.

32. Penna C, Mancardi D, Rastaldo R, Pagliaro P. Cardioprotection: a radical view Free radicals in pre and postconditioning. Biochim Biophys Acta 2009; 1787: 781-93. (Review)

33. Qin Y, Vanden Hoek TL, Wojcik K, et al. Caspase-dependent cytochrome c release and cell death in chick cardiomyocytes after simulated ischemia-reperfusion. Am J Physiol 2004; 286: H22806.

34. Shao ZH, Wojcik KR, Qin Y, et al. Blockade of caspase-2 activity inhibits ischemia/ reperfusion-induced mitochondrial reactive oxygen burst and cell death in cardiomyocytes. J Cell Death 2011; 4: 7-18.

35. Yao YT, Fang NX, Shi CX, Li LH. Sevoflurane postconditioning protects isolated rat hearts against ischemia-reperfusion injury. Chin Med J (Engl) 2010; 123: 1320-8.

36. Suzuki K, Murtuza B, Smolenski RT, et al. Overexpression of interleukin-1 receptor antagonist provides cardioprotection against ischemia-reperfusion injury associated with reduction in apoptosis. Circulation 2001; 104: I308-13.

37. Fearon WF, Fearon DT. Inflammation and cardiovascular disease: role of the interleukin-1 receptor antagonist. Circulation 2008; 117: 2577-9. (Review)

38. Siwik DA, Chang DL, Colucci WS. Interleukin-1beta and tumor necrosis factor-alpha decrease collagen synthesis and increase matrix metalloproteinase activity in cardiac fibroblasts in vitro. Circ Res 2000; 86: 1259-65.

39. Bujak M, Dobaczewski M, Chatila K, et al. Interleukin-1 receptor type I signaling critically regulates infarct healing and cardiac remodeling. Am J Pathol 2008; 173: 57-67.

40. Kiczak L, Paslawska U, Bania J, et al. Increased expression of interleukin-1beta and its novel splice variant in canine hearts with volume overload. Cytokine 2008; 44: 352-60. 